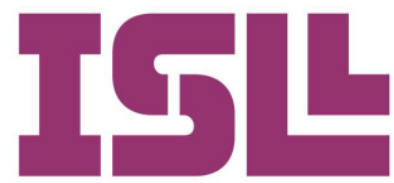

Número 6.

Julio de 2016

\section{Mirar como maestros para el desarrollo de la comprensión lectora. Blogs educativos para la competencia profesional en futuros docentes}

\section{Look as teachers to develop reading comprehension. Educational blogs for noticing in future teachers}

\author{
José Rovira-Collado \\ Dept. Innovación y Formación Didáctica \\ Universidad de Alicante
}

Pág. 58 a la 75

\section{Keywords}

Professional Noticing, Reading

Comprehension, Blogs, Language Teaching

\section{Abstract:}

Within the different lessons that develop in the courses of Primary Education and Master of Teacher Education, a principal aspect is the professional competence and teacher's noticing (Mason, 2002). Through this, the students, as future teachers, begins to interpret the teaching situations and reflects on how learning to learners in the different classrooms and subjects occurs.

One of several proposals for improving this educational look passes through the new possibilities of the Internet. In this paper we analyze some teacher's blogs as a platform for the development of this "teacher's noticing" and reading competence. We start from general examples of educational thinking and educational action blogs (Balagué and Zayas, 2007) to focus on teaching of language and literature and children's literature, spaces of academic reflection on literary education, reading comprehension and e-literacy.

\begin{abstract}
After analysis of twenty-five models we conclude that all these spaces allow us to develop the professional noticing of students to teachers of different educational stages. Reading comprehension of the content contained in these blogs allows students to demonstrate the development of the noticing.
\end{abstract}




\section{Introducción}

Entre las numerosas líneas de investigación en torno a la innovación educativa queremos centrarnos en esta ocasión en lo que conocemos como "competencia profesional" o "mirada docente" (Mason, 2002 y van Es y Sherin, 2002). En ella se analiza como el alumnado de las Facultades de Educación desarrolla una competencia específica, el noticing que consiste en identificar el aprendizaje de su alumnado y como este se desarrolla en todas las áreas de conocimiento.

\subsection{El desarrollo de la mirada profesional en el alumnado de la Facultad de Educación}

Desde los grados de Educación Infantil y Primaria hasta el Máster de Formación de Profesorado consideramos que el desarrollo de esta competencia es fundamental para el desarrollo integral del futuro docente, que toma conciencia de su evolución, a través del aprendizaje de su alumnado. Desarrollar una mirada profesional durante la formación académica es imprescindible en cualquier tipo de profesión, más allá de los distintos tipos de prácticas. En Educación, esta competencia supone identificar y promover el proceso del aprendizaje del alumnado de cualquier etapa y de cualquier asignatura.

Podemos profundizar en el concepto de la competencia "mirar profesionalmente" desde distintas perspectivas. Mason (2002) identifica cuatro características o fases en el desarrollo de capacidad docente: (1) identificar aspectos relevantes a partir de un objetivo que guía la observación (intentional noticing), (2) describir los aspectos observados (marking and recording), (3) reconocer alternativas de acción (recognizing choices), y (4) validar lo observado intentando que los otros reconozcan lo que ha sido descrito o sugerido (validating with others). Respecto al conjunto de destrezas necesarias para que los futuros docentes desarrollen esta competencia, van Es y Sherin (2002) identifican tres elementos fundamentales: (A) identificar aspectos relevantes de la situación de aprendizaje; (B) utilizar el conocimiento del contexto educativo en el que se desarrollan para reflexionar sobre las interacciones que suceden; y (C) establecer conexiones entre lo que sucede en el aula y los principios generales sobre los procesos de enseñanza aprendizaje y los contenidos de cada asignatura.

Por ejemplo, encontramos distintas investigaciones de referencia internacional en torno a la evaluación de los conocimientos matemáticos y cómo los futuros docentes aprenden a identificar estos aprendizajes (Fernández, Llinares y Valls, 2012 y 2013):

Solving problem is a relevant task in mathematics teaching. However, teachers need to understand the students' thinking in order to manage problem solving situations in classroom. Teachers' abilities to identify the mathematical key aspects in the students' thinking during problem solving are important to performance teaching for understanding. (Fernández, Llinares y Valls, 2013 p. 441). 
Esta misma línea de investigación también se aplica a las posibilidades de la tecnología y como esta nos ofrece nuevas dinámicas y nuevos escenarios de aprendizaje, como veremos más adelante. Desde enero de 2014, un grupo interdisciplinar ha desarrollado una investigación cualitativa con el alumnado de prácticas, donde se analiza el desarrollo de esta capacidad en distintas áreas, incluyendo pruebas en el ámbito de la competencia en comunicación lingüística y la competencia artística. En referencias teóricas relacionadas precedentes (Llinares y Valls, 2010 y Fernández, Llinares y Valls, 2012) también encontramos un análisis de los foros online para mostrar esta competencia docente. También el uso de narrativas escolares como instrumento de observación en el aula se ha convertido en una herramienta fundamental en el análisis de esta competencia. Ivars y Fernández $(2015,47-48)$ definen así este instrumento:

Las narrativas son historias en las que el autor relata, de manera secuencial, una serie de acontecimientos que cobran sentido para él, a través de una lógica interna. De esta manera, las narrativas de los estudiantes para maestro, en las cuales se describa lo que ellos consideran relevante sobre la enseñanza de otros maestros y sobre cómo lo que sucede en el aula apoya el aprendizaje (...) de los estudiantes, pueden ser una buena herramienta que potencie el desarrollo de la competencia docente mirar profesionalmente.

Las narrativas como descripción de una situación de aprendizaje y como herramienta de reflexión didáctica se convierten en un elemento fundamental para reconocer el desarrollo de la competencia docente. En una investigación estructurada, debemos procurar que las narrativas recojan las fases de desarrollo antes citadas.

\subsection{Hipertexto y blogs para el desarrollo de la comprensión lectora}

Entre las distintas aplicaciones de la web 2.0, los blogs se han convertido en un espacio central de innovación que nos permite crear a cualquier usuario un espacio en la red con cualquier tipo de objetivos o contenidos.

Desde esta perspectiva queremos proponer los blogs educativos como una herramienta adecuada para el desarrollo de esta competencia docente, en este caso para el área de didáctica de la lengua y la literatura, sobre todo desde el ámbito de la comprensión lectora, ya que nos encontramos ante una nueva categoría textual donde el hipertexto digital es fundamental. Además, las posibilidades de interacción a través de comentarios y redes sociales y de crear un espacio propio, nos ofrecen una nueva vía para la lectoescritura multimedia (Lara, 2009, p. 20):

El concepto de web de lecto-escritura engloba a todas aquellas aplicaciones, como son los blogs y los wikis, cuyo desarrollo tecnológico ha permitido a cualquier usuario la posibilidad de "escribir", esto es, de colgar contenidos, pensamientos, comentarios, etc. en internet de forma gratuita y sin necesidad de tener conocimientos de informática avanzados

ISL, vol. 6, 2016, págs. 58-75 ISSN: $2340-8685$
Rovira-Collado, J. (2016): Mirar como maestros para el desarrollo de la comprensión lectora. Blogs educativos para la competencia profesional en futuros docentes, Investigaciones Sobre Lectura, 6, 58-75. 
Para el adecuado desarrollo de la compresión lectora en un entorno de lectoescritura digital como son los blogs, es fundamental el concepto de hipertexto (Landow, 2009) como texto multimodal con múltiples enlaces y rutas de lectura. Estos enlaces no implican necesariamente una relación intertextual entre los elementos y pueden referirse a textos dentro del propio blog o a espacios externos. Además, la hipertextualidad digital es también multimedia y se integran en el relato otros elementos como el sonido y la imagen. Joan Campàs desarrolla el concepto de hipertexto desde sus teóricos iniciales como Vannevar Bush y Ted Nelson hasta las últimas aportaciones que convierten en realidad una nueva estructura textual: "El hipertexto es una tecnología de la información que tiene como principal característica la capacidad de emular la organización asociativa de la memoria humana" (Campàs, 2007, 41). En este sentido, el concepto de nodo y enlace son elementos centrales para la adecuada comprensión lectora de esta nueva tipología textual.

A partir de esta idea general, podemos analizar los blogs como un espacio central de la web 2.0 ya que además son los que más facilidades de edición ofrecen a los usuarios en formación. Para sus posibilidades docentes debemos asumir algunas de las clasificaciones y diferenciar entre blogs docentes y de aula (Balagué y Zayas, 2007), y la participación o no de los discentes en sus distintos niveles de escritura (entradas, comentarios, etiquetas...). La capacidad de participación social que nos ofrecen las herramientas 2.0 hace que la comprensión lectora transcienda el mero hecho de leer y nos permita conocer otras aportaciones. Es lo que conocemos como lectura social (Cordón et al., 2013) o lectura 2.0 (Lluch, 2014).

Según Jiménez (2015, p. 5), podemos diferenciar los textos digitales entre estáticos y dinámicos

Dinámicos. Son textos EDI: Enriquecidos (con enlaces multimedia, a otros textos fuera del metatexto, etc.), Discontinuos (controlados: el autor limita la red informativa, como por ejemplo un Power Point; no controlados: en los que se desconoce el número de enlaces, por ejemplo, un enlace de Wikipedia) Interactivos (primarios: que requieren la acción física del lector como ir a un enlace, interactuación mecánica, Wiki; o secundarios: que invitan a la respuesta psicológica unida a la física como contestar unas preguntas, interactuación cognitiva, iBooks Author).

Los blogs son un claro ejemplo de textos enriquecidos, discontinuos, porque dentro del elemento central de la entrada o post como eje de escritura encontramos múltiples vías de lectura e interactivos ya que el lector puede convertirse en escritor en cualquier momento, ya sea a través de comentarios, redes sociales, u otros blogs.

El concepto de hipertexto 2.0 tiene una clara proyección didáctica (Mendoza, Arbonés y Muñoz, 2015) respecto a la compresión lectora y la competencia literaria. Soria 
Andurell (2015) nos muestra una investigación concreta de cómo el hipertexto influye en la compresión lectora del alumnado de Educación Primaria. Interpretar cómo se produce ese aprendizaje a través de una lectura digital debe ser otro eslabón de la competencia docente del futuro profesorado. El desarrollo de la comprensión lectora en el entorno digital debe estar relacionado obligatoriamente con el desarrollo de la competencia lectoliteraria. Por lo tanto, la presencia de la literatura infantil y juvenil en la web social y el concepto de LIJ 2.0 (Llorens y Rovira, 2012, Rovira, 2015) son también herramientas para comprender esta blogosfera.

Son los blogs, por lo tanto, un espacio idóneo de participación y de desarrollo de la competencia lectoliteraria para el alumnado desde las primeras etapas y una herramienta para practicar la "mirada docente" del alumnado para maestro.

\section{Metodología: Análisis de blogs docentes}

Una vez citadas las posibilidades docentes de los blogs para el desarrollo de la comprensión lectora y la competencia profesional, pasamos a describir distintos espacios de referencia que pueden servir como modelo a nuestro alumnado para maestro, ya sea de Infantil, Primaria o Secundaria. Los participantes de esta investigación son los veinticinco espacios descritos y las personas relacionadas con ellos. Algunas de estas bitácoras tienen una trayectoria de más de diez años y otras son la evolución de anteriores espacios. El procedimiento para este análisis ha sido la descripción de los blogs como instrumento idóneo para el desarrollo de la comprensión lectora y la competencia “mirar profesionalmente". Se establecen seis variables de partida para la clasificación general de los modelos, pero como veremos luego, existen muchos elementos en común entre los distintos ejemplos. En la discusión se ofrece una tabla globalizadora que recoge los espacios analizados. Partiremos desde las propuestas más generales a las concretas de didáctica de la lengua y la literatura.

\subsection{Blogs docentes generalistas}

En primer lugar, incluimos espacios de referencia internacional fundamentales para conocer el medio digital.

1. Jose Luis Orihuela http://www.ecuaderno.com/. El eCuaderno. Pistas, noticias y enlaces sobre los medios y la red, creado en 2002, es posiblemente el primer espacio destacado en la blogosfera educativa, tanto por tradición como por calidad y cantidad de los contenidos. Fruto de esta práctica temprana encontramos manuales obligatorios que ya se han convertido en clásicos como La revolución de los blogs (2006) hasta propuestas más actuales como Mundo Twitter (2011), donde analiza otro espacio de relevancia en la comunicación digital como es el microblogging.

ISL, vol. 6, 2016, págs. 58-75 ISSN: $2340-8685$
Rovira-Collado, J. (2016): Mirar como maestros para el desarrollo de la comprensión lectora. Blogs educativos para la competencia profesional en futuros docentes, Investigaciones Sobre Lectura, 6, 58-75. 
2. Enrique Dans http://www.enriquedans.com/. Creado en febrero de 2003, nos encontramos con otro espacio central en la difusión de la cultura digital en nuestro país. Con actualizaciones diarias y una edición bilingüe se ha convertido en un espacio de obligada consulta para conocer los últimos avances digitales. Aunque es muy importante, este espacio está sometido en ocasiones a distintas polémicas ya que su autor escribe sobre cualquier tema relacionado con las TIC. Es el caso de las aportaciones en torno a la nueva propuesta en torno a la enseñanza de la escritura que Finlandia lanzó a finales de 2014 (http://www.enriquedans.com/2014/11/escribir-a-mano-es-del-siglo-pasado.html y http://www.enriquedans.com/2014/12/caligrafia-educacion-y-futuro-examinando-losargumentos.html). Aunque son entradas muy interesantes y bien documentadas, y compartimos en parte la perspectiva del autor, se podrían anotar algunas carencias desde el ámbito de didáctica de la lengua y la literatura.

3. Dolors Reig http://www.dreig.eu/caparazon/ es una de las mayores especialistas en las transformaciones que Internet ha supuesto para la educación. En 2007 lanzó El Caparazón como un espacio de reflexión sobre "social media, Internet, psicología, educación, redes sociales, innovación y creatividad". Aunque es una web muy completa, respeta muchas de las características de los blogs. En el diseño de la página encontramos distintos widgets que nos llevan a distintas secciones u otras webs. También nos sirven para recoger las publicaciones de la autora o los reconocimientos a este espacio. El concepto de Aprendizaje Social y en Abierto (Open Social Learning) es imprescindible para entender los objetivos y el alcance de este blog.

\subsection{El reto de las 365 entradas}

Al igual que en el caso anterior de Enrique Dans, encontramos otros espacios que nos ofrecen diariamente una entrada. Esta dinámica de utilizar el blog como un diario es muy interesante porque crean expectativas a los lectores y fidelizan a la audiencia. Diario personal o diario de aprendizaje, esta práctica implica una dedicación al blog muy importante, que se suele ver recompensada por un gran número de seguidores. Por ejemplo, podemos citar:

1. Jordi Martí http://www.xarxatic.com/. Aunque existe una "familia" de autores que colabora con esta página, es Jordi Martí el principal artífice de este blog con reflexiones constantes sobre la educación en general. Aunque el diseño es de una página completa con muchos espacios y el apoyo de otros medios sociales, lo seguimos considerando un blog porque en sus entradas diarias hay una conversación constante a través de los comentarios, que convierten al blog en una matriz de foros educativos con múltiples debates. También encontramos una característica propia de estos enlaces: el blogroll o lista de enlaces, http://www.xarxatic.com/edublogs/ que nos permite adentrarnos en una blogosfera educativa con muchos espacios con intereses similares a los del autor.

2. Santiago Moll http://justificaturespuesta.com/ Es uno de los espacios más jóvenes porque publicó su primer artículo un 22 de abril de 2012. Sin embargo, se ha convertido en un espacio a tener en cuenta que además tiene legión de seguidores en las redes sociales. Al 
igual que el anterior, es un espacio de reflexión educativa con infinidad de recursos para el docente y muchas de sus aportaciones nos servirán para el desarrollo de la mirada profesional. Consejos de actuación docente, listados de actividades y otras muchas entradas parecen creadas específicamente para mejorar nuestra capacidad docente y por lo tanto, también la mirada profesional. El autor además difunde cada entrada a través de múltiples herramientas sociales como los grupos de Facebook.

\subsection{La voz del alumnado}

Desde nuestra experiencia como docente, descubrir que el alumnado crea su propio espacio a partir de prácticas en el blog de aula es una constatación del interés por la herramienta, que se utiliza en estos casos con objetivos docentes e investigadores. A continuación, citamos primer cuatro ejemplos, pero nos consta que otros muchos exalumnos han adoptado la herramienta como instrumento didáctico.

1. Jesús Vidal Martín-Toledano nos ofrece dos propuestas de actuación http://aprendizaje20.blogspot.com.es/ (blog dedicado a tecnología educativa) y https://abibliotecasecretadebrucewayne.wordpress.com/ (bitácora recientemente migrada desde Blogger centrada en la narración gráfica, principalmente el cómic); Luis Felipe Güemes http://elatlasdelafantasia.blogspot.com.es/ (con un blog como apoyo a su tesis doctoral); e Ignacio Ballester http://otaler.blogspot.com.es/ (sobre literatura en general e investigación filológica) son tres ejemplos cercanos de exalumnos, que después de una práctica docente con blogs, crean un espacio propio para proponer sus ideas y trabajos. La mirada docente está presente en todos ellos.

Posteriormente tenemos el concepto de Metablog, donde un docente puede crear un espacio que sirva de nodo central a blogs de su alumnado. Como, por ejemplo:

1. Ricardo Fernández http://practicummagisterio.blogspot.com.es/. Siendo el periodo de prácticas en las distintas titulaciones de formación docente una etapa idónea para el desarrollo de la mirada profesional, encontramos profesores que recogen en blogs todo el trabajo de su alumnado en las prácticas para ofrecérselo a otras personas, como es el caso de Diario de alumnos de Prácticas. El blog puede tener pocas entradas, pero enlaza con los blogs del alumnado en prácticas de los últimos cinco años, mostrando la reflexión docente de cada persona. Aunque no se recoge en los blogs recogidos, recomendar al alumnado en prácticas la escritura de una entrada cada día favorece su uso como diario de aprendizaje, otra muestra de escritura para la competencia docente.

2. También hay metablogs mixtos, docentes e investigadores, que además de recoger la práctica investigadora, enlazan con otros blogs didácticos. Por ejemplo, en Estudiando la LIJ en la web social, http:/www.literaturainfantilyjuvenileninternet.blogspot.com.es/ de José Rovira, encontramos enlazados en la barra superior los blogs de distintas asignaturas

ISL, vol. 6, 2016, págs. 58-75 ISSN: $2340-8685$
Rovira-Collado, J. (2016): Mirar como maestros para el desarrollo de la comprensión lectora. Blogs educativos para la competencia profesional en futuros docentes, Investigaciones Sobre Lectura, $6,58-75$. 
generados por el alumnado universitario a lo largo de los últimos ocho años con un corpus de más de mil quinientas entradas.

\subsection{Docencia e investigación}

Llegando a la práctica docente diaria, queremos citar ejemplos de profesionales que utilizan el blog como plataforma digital para sus numerosos proyectos educativos:

1. $\mathrm{M}^{\mathrm{a}}$ Carmen Devesa, http://mcarmendevesa.blogspot.com.es/ nos propone TIC $y$ Aprendizaje, creado en 2010, para recoger todos los proyectos realizados con su alumnado o en colaboración con otros centros escolares. Recursos, presentaciones, herramientas 2.0 y secciones para familias y profesorado son los espacios en los que se divide esta bitácora. Por ejemplo, en las últimas entradas encontramos referencias al ABP, el Aprendizaje Basado en Proyectos, una metodología que está cogiendo mucha fuerza en los últimos tiempos. A través de sus ejemplos se puede confirmar que la integración de la tecnología se puede realizar desde los primeros niveles educativos.

2. Conchita López http://blogmaniacosunidos.blogspot.com.es/ Blogmaniacos es otro ejemplo de maestra que utiliza el blog para compartir sus actividades en el aula, donde el protagonismo principal es el de su alumnado. Aunque parecía que la autora iba a abandonar el espacio después de su jubilación, sigue publicando alguna entrada cada cierto tiempo, con entradas que demuestran que la mirada profesional va más allá de la docencia reglada. Entre las distintas secciones podemos identificar casi todos los premios docentes o reconocimientos a prácticas docentes innovadoras, como por ejemplo las "Buenas prácticas" del Ministerio de Educación. Sus propuestas no se circunscriben al alumnado de Educación Primaria. Por ejemplo, a través de su experiencia surgió el proyecto Uniblogmaniacos, http://www.educacontic.es/blog/proyecto-uniblogmaniacos donde su grupo de clase interaccionaba con distintos grupos de alumnado universitario a través de la evaluación de vídeos educativos creados para esta clase. La perspectiva de este proyecto es otro ejemplo del interés por trabajar la mirada docente en el aula universitaria.

3. Mercedes Ruiz https://londonphd.wordpress.com/; es tal la relevancia de Blogmaniacos, que podemos citar un blog de investigación centrado en la anterior experiencia. La autora, conocida como @londones en Twitter diseñó este Blog de campo de una doctoranda, para articular la investigación sobre las importantes cualidades educativas del anterior espacio. Dentro de la competencia "Mirar profesionalmente", la reflexión teórica y metodológica en una tesis doctoral sobre educación es uno de los textos de mayor complejidad que se puede ofrecer. La comprensión lectora de los textos incluidos en la propia tesis (en prensa), así como de las entradas del blog supone la concreción de esa mirada docente. 


\subsection{Espacios de referencia académica}

Frente a los grupos anteriores, encontramos los espacios de referencia académica. Aunque en los precedentes la cantidad de información suele ser mayor, en estos hay siempre un apoyo científico en las entradas. Por este motivo, muchos de ellos tienen una publicación menos frecuente, ya que cada entrada conlleva una investigación mayor. Con el desarrollo de las herramientas 2.0 para la promoción de la producción científica (redes sociales académicas, gestores de referencias, repositorios institucionales...), el blog se convierte en un espacio propio con incontables posibilidades de edición para cada investigador. Estos espacios ya no se limitan simplemente a la práctica docente, sino a la reflexión académica sobre muchos aspectos pedagógicos. En nuestro análisis, queremos destacar:

1. Jordi Adell http://elbonia.cent.uji.es/jordi/. Más que un blog podemos considerarlo como el espacio personal en la web de la Universidad Jaume I de uno de los mayores especialistas en tecnología educativa en nuestro país. Sin embargo, el tono y la redacción de las entradas son muy cercanos a los blogs, aunque siempre con temas fundamentales de la práctica docente. Este tipo de escritos está enfocado a especialistas y si nuestro alumnado puede interpretar estos trabajos y sumarlos a su formación personal, es que ha desarrollado en gran parte su mirada profesional. Como mucho de los espacios trabajados, es la evolución de anteriores webs o blogs y el propio autor nos ofrece el enlace a estos espacios de experimentación inicial.

2. Tíscar Lara http://tiscar.com/. Otro espacio que ha sido fundamental para nuestra concepción de los blogs y sus posibilidades educativas para la alfabetización digital (Lara, 2009) es este espacio. En sus entradas podemos encontrar muchas claves del desarrollo tecnológico de la educación en la actualidad, siempre aportando referencias de calidad y estudios internacionales. Desde el Mobile Learning a las posibilidades de blogs y wikis para la didáctica de las lenguas, encontramos múltiples referencias académicas que nos indican hacia dónde se dirigen la tecnología educativa en los próximos años.

3. David Álvarez http://e-aprendizaje.es/. Su autor, conocido como @balhisay en Twitter, es otro de los referentes académicos en la introducción de nuevas tendencias en tecnología educativa, como por ejemplo el aprendizaje social. Al igual que los anteriores, su página está llena de recursos y secciones, pero las novedades periódicas nos permiten considerarlo un blog. Dentro de la nueva concepción del aprendizaje conectado, este espacio y su autor son referencias imprescindibles en la configuración del Espacio Personal de Aprendizaje (PLE Personal Learning Environment) de cualquier futuro docente.

4. Fernando Trujillo http://fernandotrujillo.es/ Aunque pertenece al área de Didáctica de la lengua y la literatura, el profesor Trujillo es uno de las referencias principales en tecnología educativa en nuestro país. Al igual que muchos de los casos trabajados, esta página proviene de la experiencia al frente de un blog docente personal http://deestranjis.blogspot.com.es/. Este hecho, que se repite en muchos de los ejemplos citados, muestra la relevancia de los blogs como primer espacio de publicación digital de

ISL, vol. 6, 2016, págs. 58-75 ISSN: $2340-8685$
Rovira-Collado, J. (2016): Mirar como maestros para el desarrollo de la comprensión lectora. Blogs educativos para la competencia profesional en futuros docentes, Investigaciones Sobre Lectura, $6,58-75$. 
muchos docentes, que posteriormente se animarán a crear páginas más complejas. Su web recoge también el currículum personal que nos permite acceder a las publicaciones, facilitando la difusión de su investigación, así como muchas presentaciones y vídeos de participaciones en jornadas.

2.6. Blogosfera de Literatura Infantil y Juvenil (LIJ) y de Didáctica de la Lengua y la Literatura $(D L L)$

Centrándonos en el área de conocimiento de Didáctica de la lengua y literatura, en los últimos años se han desarrollado distintas investigaciones sobre las posibilidades de estos espacios tanto para la difusión de la LIJ en la web 2.0 (Rovira, 2011a y 2015) como para la práctica docente en DLL (Rovira, 2011b y Llorens y Rovira, 2012). Como anexo se ofrece un listado de veinticinco espacios de referencia, algunos incluidos en este análisis, que nos pueden servir como modelo de buenas prácticas para la enseñanza de la lengua y la literatura en distintos niveles.

Encontramos distintas posibilidades para clasificar las bitácoras de LIJ (García y Rubio, 2013) que nos permiten navegar en un océano inmenso de blogs. Nombres como los de Jorge Gómez Soto, Dolors Insa, Darabuc o Román Belmonte son fundamentales para conocer las distintas posibilidades de esta variedad de blogs didácticos y de LIJ, que están completamente ligados al ámbito de la comprensión lectora. A continuación, solamente vamos a citar distintos ejemplos como modelos imprescindibles de miradas docentes e investigadoras desde el área de DLL.

1. Toni Solano http://www.repasodelengua.com/. El profesor Solano es posiblemente uno de los mejores ejemplos de integración de la tecnología en el aula de Educación Secundaria de lengua y literatura. Su blog funciona como bitácora docente, pero además nos enlaza a muchos de sus proyectos, que han recibido múltiples reconocimientos como modelos de actuación en el aula. De esta experiencia surgen además otros blogs como http://detextos.blogspot.com.es/ Sitio de recursos TIC para profesores, que le permiten ampliar los espacios de actuación, los cursos y los proyectos.

2. Eduardo Larequi http://www.labitacoradeltigre.com/. Otro caso similar es el del profesor Larequi, cuyo blog es uno de los espacios pioneros de nuestra área. Con más de ochocientas entradas entre 2006 y 2013, esta reflexión pedagógica o mirada docente ha dado lugar a otros espacios como http://www.lenguaensecundaria.com/, un referente para el área de lengua y literatura en el Máster de Formación del Profesorado.

3. Pep Hernández http://www.apuntesdelengua.com/blog/. Con esta web nos encontramos también ante un espacio magnífico como apoyo a la docencia pero sobre todo con muchas entradas que favorecen esas reflexiones docentes que comparte su autor. Presentaciones de diapositivas, vídeos, mapas mentales y otros materiales multimedia están incluidos entre los múltiples recursos de este blog que es un ejemplo a seguir para cualquier docente de lengua y literatura. 
4. Literaturas exploratorias https://iteraturasexploratorias.com/. La doctora Celia Turrión creó este espacio en 2011 como apoyo a su investigación sobre la Literatura Infantil y Juvenil Digital. La bitácora como espacio de apoyo y difusión de una tesis doctoral es común en otros ejemplos citados. Posteriormente se ha sumado al proyecto el investigador Lucas Ramada. Ambos promueven estudios sobre las transformaciones digitales de la lectura y en la LIJ a través de la colaboración con el grupo de investigación de la Universitat Autònoma de Barcelona Gretel http://www.gretel.cat/.

5. Felipe Zayas http://www.fzayas.com/darlealalengua/. Con Felipe Zayas nos encontramos posiblemente con el maestro de maestros en el uso de blogs docentes (2008). Como él mismo nos cuenta, ha pasado por muchas plataformas y además ha participado en varios proyectos colaborativos como el clásico El Tinglado http://www.tinglado.net/?category=blogsyuser=felipezayas hasta el más reciente Centro Virtual Leer.es http://blog.leer.es/. La experiencia docente de su autor es visible en todas las entradas. Al hablarnos de la última etapa de su blog nos plantea las siguientes líneas de trabajo, que puede relacionarse directamente con los objetivos de esta investigación:

- La información sobre las propias experiencias de aula en la enseñanza de la lengua y de la literatura, así como la reflexión sobre el uso didáctico de los blogs de aula y, en general, de los recursos TIC.

- Las reflexiones sobre los temas típicos de la didáctica de la lengua y la literatura: la competencia lectora, la composición de textos, la educación literaria, la reflexión gramatical, etc.

- La reflexión sobre los blogs como nuevas formas de comunicación. (Zayas, 2013)

6. Centro Virtual Leer.es El proyecto Leer.es http://leer.es/ es, desde su creación, un espacio fundamental para la reflexión sobre la evolución de la lectura en el siglo XXI. Aunque el espacio del blog ha desaparecido como espacio de noticias periódicas dentro de la web, podemos encontrar todas las novedades del blog a través del repositorio de Educalab del Centro Nacional de Innovación e Investigación Educativa (CNIIE) http://blog.educalab.es/cniie/category/competencias-basicas/leeres/. Este es el único espacio oficial que hemos incluido en esta clasificación, ya que en la publicación del blog participan distintos especialistas en el ámbito de la lectura.

7. Gemma Lluch http://www.gemmalluch.com. Este espacio es seguramente uno de los principales modelos de actuación para difundir la investigación académica en torno a la literatura infantil y juvenil y por supuesto también a los distintos aspectos de la comprensión lectora. Aunque también es una web con dominio personalizado, la riqueza son sus entradas periódicas que además están muchas veces entrelazadas entre sí, en un sistema de recomendación propio de la lectura social y del hipertexto digital, que convierten a este blog en un manual docente con infinitas rutas de lectura. Las entradas están perfectamente elaboradas y muchas veces vienen acompañadas con la noticia, el enlace o directamente con el acceso a una publicación científica de la autora. Como en todos los anteriores casos, la labor de este blog viene acompañada por una constante actividad en las redes sociales que permite ampliar el campo de actuación del blog y de la autora, cuyas publicaciones cuentan con miles de seguidores en todo el mundo.

ISL, vol. 6, 2016, págs. 58-75 ISSN: $2340-8685$
Rovira-Collado, J. (2016): Mirar como maestros para el desarrollo de la comprensión lectora. Blogs educativos para la competencia profesional en futuros docentes, Investigaciones Sobre Lectura, $6,58-75$. 


\section{Discusión}

Se han distribuido los distintos espacios en seis categorías (1. Blogs docentes generalista, 2. El reto de las 365 entradas, 3. La voz del alumnado, 4. Docencia e investigación, 5 Espacios de referencia académica y 6. Blogosfera de LIJ y de DLL), que nos permiten partir de los espacios más conocidos y generales a modelos concretos dentro del ámbito de la didáctica de la lengua y la literatura. Además, encontramos desde espacios clásicos, que se lanzaron en la década anterior como espacios de innovación, a propuestas más recientes que nos permiten constatar la vigencia de la herramienta. La siguiente tabla engloba los principales datos de la investigación según el orden de descripción anterior:

\begin{tabular}{|c|c|c|c|c|}
\hline Nombre & Dirección & Autor/a & $\begin{array}{l}\text { Creado } \\
\text { en* }\end{array}$ & Contenido \\
\hline eCuaderno & http://www.ecuaderno.com/ & $\begin{array}{l}\text { Jose Luis } \\
\text { Orihuela }\end{array}$ & 2002 & $\begin{array}{l}\mathrm{D}, \mathrm{I}, \mathrm{ID}, \mathrm{PU} \\
\mathrm{TE}\end{array}$ \\
\hline Enrique Dans & http://www.enriquedans.com/ & Enrique Dans & 2003 & $\begin{array}{l}\text { D, I, ID, PU, } \\
\text { SE, TE }\end{array}$ \\
\hline El Caparazón & http://www.dreig.eu/caparazon/ & Dolors Reig & 2007 & $\begin{array}{l}\text { D, I, ID, PU, } \\
\text { SE, TE }\end{array}$ \\
\hline Xarxatic & http://www.xarxatic.com/ & $\begin{array}{l}\text { Jordi Martí (y } \\
\text { otros }\end{array}$ & $\begin{array}{l}\text { No } \\
\text { aparece }\end{array}$ & $\begin{array}{l}\mathrm{D}, \mathrm{I}, \mathrm{ID}, \mathrm{SE} \\
\mathrm{TE}\end{array}$ \\
\hline $\begin{array}{l}\text { Justifica tu } \\
\text { respuesta }\end{array}$ & http://justificaturespuesta.com/ & Santiago Moll & 2012 & $\begin{array}{l}\mathrm{D}, \mathrm{I}, \mathrm{ID}, \mathrm{SE} \\
\mathrm{TE}\end{array}$ \\
\hline Aprendizaje 2.0 & $\underline{\text { http://aprendizaje2- }}$ & $\begin{array}{l}\text { Jesús Vidal } \\
\text { Martín-Toledano }\end{array}$ & 2013 & A, ID, TE. \\
\hline $\begin{array}{l}\text { Biblioteca } \\
\text { secreta B } \\
\text { Wayne }\end{array}$ & $\begin{array}{l}\text { https://labibliotecasecretadebrucew } \\
\text { ayne.wordpress.com/ }\end{array}$ & $\begin{array}{l}\text { Jesús Vidal } \\
\text { Martín-Toledano }\end{array}$ & 2014 & A, LIJ, O. \\
\hline $\begin{array}{l}\text { Atlas de la } \\
\text { Fantasía }\end{array}$ & $\begin{array}{l}\underline{\text { http://elatlasdelafantasia.blogspot.c }} \\
\underline{\text { om.es/ }}\end{array}$ & Luis F. Güemes & 2014 & A, I, LIJ. \\
\hline $\begin{array}{l}\text { Retales } \\
\text { coloquiales }\end{array}$ & http://otaler.blogspot.com.es/ & Ignacio Ballester & 2012 & $\begin{array}{l}\text { A, D, I, DLL, } \\
\text { LIJ. }\end{array}$ \\
\hline $\begin{array}{l}\text { Estudiando LIJ } \\
\text { en web social }\end{array}$ & $\underline{\text { http://www.literaturainfantilyjuveni }}$ & $\begin{array}{l}\text { José Rovira } \\
\text { Collado }\end{array}$ & 2008 & $\begin{array}{l}\text { A, D, DLL, } \\
\text { ME, LIJ, PU. }\end{array}$ \\
\hline $\begin{array}{l}\text { Diario de } \\
\text { alumnos de } \\
\text { Prácticas }\end{array}$ & $\begin{array}{l}\text { http://practicummagisterio.blogspot } \\
\text {.com.es/ }\end{array}$ & $\begin{array}{l}\text { Ricardo } \\
\text { Fernández }\end{array}$ & 2009 & A, D, SE, O. \\
\hline $\begin{array}{l}\text { TICy } \\
\text { Aprendizaje }\end{array}$ & $\begin{array}{l}\text { http://mcarmendevesa.blogspot.com } \\
\text {.es/ }\end{array}$ & $\begin{array}{l}\text { M. Carmen } \\
\text { Devesa }\end{array}$ & 2010 & A, D, ID, TE. \\
\hline Blogmaniacos & $\begin{array}{l}\text { http://blogmaniacosunidos.blogspot } \\
\text { com.es/ }\end{array}$ & Conchita López & 2008 & $\begin{array}{l}\text { A, D, ID, LIJ, } \\
\text { TE, O. }\end{array}$ \\
\hline $\begin{array}{l}\text { Blog de campo } \\
\text { de una } \\
\text { doctoranda }\end{array}$ & $\underline{\text { https://londonphd.wordpress.com/ }}$ & Mercedes López & 2011 & $\mathrm{D}, \mathrm{I}, \mathrm{ID}, \mathrm{O}$. \\
\hline$E d \& T e c$ & $\underline{\text { http://elbonia.cent.uji.es/jordi/ }}$ & Jordi Adell & $\begin{array}{l}\text { No } \\
\text { aparece }\end{array}$ & $\begin{array}{l}\text { D, I, ID, PU, } \\
\text { SE, TE. }\end{array}$ \\
\hline Tíscar & http://tiscar.com/ & Tíscar Lara & 2004 & $\begin{array}{l}\text { D, DLL, I, ID, } \\
\text { PU, TE, O. }\end{array}$ \\
\hline e-aprendizaje & $\underline{\text { http://e-aprendizaje.es/ }}$ & David Álvarez & 2010 & $\begin{array}{l}\text { I, ID, PU, SE, } \\
\text { TE, O. }\end{array}$ \\
\hline
\end{tabular}

Rovira-Collado, J. (2016): Mirar como maestros para el desarrollo de la comprensión lectora. Blogs educativos para la competencia profesional en futuros docentes, Investigaciones Sobre Lectura, $6,58-75$. 


\begin{tabular}{|c|c|c|c|c|}
\hline $\begin{array}{l}\text { Fernando } \\
\text { Trujillo }\end{array}$ & http://fernandotrujillo.es/ & Fernando Trujillo & 2006 & $\begin{array}{l}\text { D, DLL, I, ID, } \\
\text { PU, SE, TE. }\end{array}$ \\
\hline $\begin{array}{l}\text { Re (paso) de } \\
\text { lengua }\end{array}$ & http://www.repasodelengua.com/ & Toni Solano & 2006 & $\begin{array}{l}\text { A, D, DLL, } \\
\text { ID, SE. }\end{array}$ \\
\hline $\begin{array}{l}\text { La bitácora del } \\
\text { Tigre }\end{array}$ & http://www.labitacoradeltigre.com/ & Eduardo Larequi & 2005 & $\begin{array}{l}\text { D, DLL, ID, } \\
\text { SE, TE. }\end{array}$ \\
\hline $\begin{array}{l}\text { Apuntes de } \\
\text { Lengua }\end{array}$ & $\begin{array}{l}\text { http://www.apuntesdelengua.com/bl } \\
\underline{\mathrm{og} /}\end{array}$ & Pep Hernández & $\begin{array}{l}\text { No } \\
\text { aparece }\end{array}$ & $\begin{array}{l}\text { A, D, DLL, } \\
\text { ID, LIJ, ME, } \\
\text { PU, TE. }\end{array}$ \\
\hline $\begin{array}{l}\text { Literaturas } \\
\text { exploratorias }\end{array}$ & https://literaturasexploratorias.com/ & $\begin{array}{l}\text { Celia Turrión y } \\
\text { Lucas Ramada }\end{array}$ & 2011 & I, ID, LIJ, TE. \\
\hline $\begin{array}{l}\text { Darle a la } \\
\text { lengua }\end{array}$ & $\underline{\text { http://www.fzayas.com/darlealaleng }}$ & Felipe Zayas & 2006 & $\begin{array}{l}\text { D, DLL, I, ID, } \\
\text { PU, SE. }\end{array}$ \\
\hline $\begin{array}{l}\text { Centro Virtual } \\
\text { Leer.es }\end{array}$ & $\begin{array}{l}\overline{\mathrm{http}: / / \text { leer.es/ }} \\
\mathrm{http://blog.educalab.es/cniie/categor} \\
\text { y/competencias-basicas/leeres/ }\end{array}$ & AAVV & 2013 & $\begin{array}{l}\text { A, D, DLL, I, } \\
\text { ID, LIJ, ME, } \\
\text { PU, SE. }\end{array}$ \\
\hline Gemma Lluch & http://www.gemmalluch.com & Gemma Lluch & 2011 & $\begin{array}{l}\text { D, DLL, I, } \\
\text { LIJ, ME, PU, } \\
\text { SE, TE. }\end{array}$ \\
\hline
\end{tabular}

Respecto al contenido se han seleccionado los siguientes ítems para la concreción del análisis: (A) Alumnado; (D) Docencia; (DLL) Didáctica Lengua y Literatura; (I) Investigación; (ID) Innovación Didáctica; (LIJ) Literatura Infantil y Juvenil (ME) Metablog; (PU) Publicaciones; (SE) Sistema Educativo; (TE) Tecnología Educativa; (O) Otros temas.

Como vemos, los últimos espacios son los que más contenidos recogen, cuando se supone que los primeros son los más generalistas y más veteranos. Esto es debido al propio diseño de la investigación, ya que los últimos ejemplos son los que están más relacionados con nuestra área de conocimiento y por lo tanto tienen más elementos en la descripción de contenidos. En una descripción desde otra perspectiva y con otros ítems, los primeros espacios recibirían mayor concreción en los contenidos.

Un elemento fundamental para la concepción del blog es la publicación periódica, que lo convierte en un texto dinámico. En algunos blogs no aparece indicada la fecha de la primera entrada ni un archivo estructurado. También hay espacios herederos de experiencias anteriores y que son estructuras superiores a un blog. Todos ellos son un relato educativo donde también podemos identificar la evolución de la mirada docente de los propios autores. La edición individual propia de la mayoría de los blogs analizados también supone una obsolescencia programada, relacionada directamente con las posibilidades e intereses de sus autores. La vitalidad de cada uno de ellos es relativa ya que proyectos con muchas entradas han podido quedar abandonados y otros que tienen ahora poca presencia pueden crecer en poco tiempo, transformarse en otros espacios o incluso desaparecer.

ISL, vol. 6, 2016, págs. 58-75 ISSN: $2340-8685$
Rovira-Collado, J. (2016): Mirar como maestros para el desarrollo de la comprensión lectora. Blogs educativos para la competencia profesional en futuros docentes, Investigaciones Sobre Lectura, $6,58-75$. 
En cualquier selección de blogs siempre corremos el riesgo de dejar fuera espacios principales o incluir algunos espacios por una interpretación subjetiva y personal. Como una escritura personal e individualizada, estos blogs recogen gran parte del desarrollo profesional de distintas personas y es interesante reconocer distintas fases en este. Por eso consideramos relevante la inclusión de espacios de investigación doctoral o de alumnado. Pero además, la mayoría de estos espacios incluye una lista de enlaces (Blogroll) o secciones específicas que nos llevan a otros muchos espacios de características o intereses similares. En este sentido la selección no pretende ofrecer un panorama global de las posibilidades, sino un recorrido de experiencias que favorezcan el desarrollo de la competencia profesional. Este es el eje de la investigación junto a la importancia de los blogs para la comprensión lectora entre las nuevas textualidades digitales.

Aunque en la actualidad existen otros espacios centrales en la web social como son las redes sociales (de distinta tipología como Facebook, Linkedin, o Academia.edu) o el microblogging (Twitter), los blogs continúan siendo el espacio de publicación central para una reflexión estructurada. La navegación adecuada a través de todos ellos mediante los múltiples hiperenlaces es una capacidad imprescindible para la comprensión lectora de la ingente cantidad de información que nos ofrecen. Además, la facilidad de edición nos permite generar espacios propios desde las primeras etapas educativas... o desde las primeras experiencias profesionales como docentes.

\subsection{Conclusiones}

Como hemos visto en estos veinticinco ejemplos, las perspectivas son múltiples y tan variadas como puede ser la práctica docente. La "mirada profesional" es intrínseca a cada uno de los espacios citados que van desde la reflexión diaria, y en algunos casos, superficial, pero que favorece el flujo de información y el debate, hasta la investigación académica. Por este motivo, algunos de los espacios analizados no tienen la misma vitalidad que otros, pero mantienen el número de seguidores y el interés de la comunidad docente. Encontramos múltiples modelos, algunos más científicos frente a otros que incluso podrían considerarse más comerciales porque tienen herramientas para monetizar las visitas, aunque estos elementos sirven para mantener el espacio. Todos ellos son espacios muy útiles para compartir la práctica docente e investigadora y hablar con otros internautas sobre cuestiones pedagógicas.

También hemos citado alguna práctica de personas recientemente egresadas que empiezan en el mundo educativo. Ellos también nos muestran su mirada docente y la próxima meta de esta investigación será analizar blogs docentes de nuestro alumnado y como se refleja en ellos la mirada profesional. La completa comprensión por parte de nuestro alumnado de los contenidos que se recogen en muchas de estas entradas puede ser un criterio evidente para demostrar el desarrollo de la mirada docente en el área de Didáctica de la lengua y la literatura. 
Al ser un blog un medio de comunicación escrito, que además permite la integración de elementos audiovisuales, nos permite el trabajo de la comprensión lectora en todas las etapas educativas y el futuro profesorado puede aprovecharlos para trabajarla en el aula.

Al igual que la competencia comunicativa y la comprensión lectora son destrezas que van aumentando a lo largo de toda la vida, la competencia digital y la mirada profesional también van incrementándose según vamos creciendo e incorporando nuevas experiencias en la formación personal y académica.

\section{Bibliografía}

Balagué, F. y Zayas, F. (2007). Usos educatius dels blogs. Recursos, orientacions $i$ experiències per a docents. Barcelona: UOC.

Campàs, J. (2007) El hipertexto. Barcelona: UOC.

Cordón García, J.A. et al. (2013). Social Reading: Platforms, Aplications, Clouds and Tags. Oxford, Chandos Publishing.

Fernández C., Llinares S., y Valls J. (2012). Learning to notice students' mathematical thinking through on-line discussions. ZDM Mathematics Education DOI 10.1007/s11858-012-0425-y.

Fernández C., Llinares S., y Valls J. (2013). Primary school teacher's noticing of students' mathematical thinking in problem solving. The Mathematics Enthusiast, 10, nos.1y2, 441-468.

García Rodríguez, A. y Rubio González, E. (2013). Un paseo por la blogosfera de la literatura infantil y juvenil española: de los blogs "lijeros" a Facebook. Puntos de Encuentro. Los primeros 20 años de la Facultad de Traducción y Documentación de la Universidad de Salamanca. Salamanca: Universidad de Salamanca, 51-72.

Ivars, P. y Fernández, C. (2015). Aprendiendo a mirar profesionalmente el pensamiento matemático de los estudiantes en el contexto de las prácticas de enseñanza. El papel de las narrativas. ENSAYOS, Revista de la Facultad de Educación de Albacete, 30(1), 45-54. Recuperado el 1 de abril, 2016 de http://www.revista.uclm.es/index.php/ensayos.

Jiménez-Pérez, E. (2015) La influencia de las TIC en la forma de leer: Tipología de textos digitales. Álabe (en prensa), Recuperado el 1 de abril, 2016 de https://www.researchgate.net/publication/270575717 LA INFLUENCIA DE LAS TIC EN LA FORMA DE LEER TIPOLOGIA DE TEXTOS DIGIT ALES.

ISL, vol. 6, 2016, págs. 58-75 ISSN: $2340-8685$
Rovira-Collado, J. (2016): Mirar como maestros para el desarrollo de la comprensión lectora. Blogs educativos para la competencia profesional en futuros docentes, Investigaciones Sobre Lectura, $6,58-75$. 
Landow, G.P. (2009). Hipertexto 3.0. La teoría crítica y los nuevos medios en una época de globalización. Barcelona, Paidós, 2006.

Lara, T. (2009). Alfabetizar en la cultura digital. En AA.VV. La competencia digital en el área de Lengua. Madrid: Octaedro, 9-38.

Llinares, S., y Valls, J. (2010). Prospective primary mathematics teachers' learning from on-line discussions in a virtual videobased environment. Journal of Mathematics Teachers Education, 13, 177-196.

Llorens García, R. F. y Rovira-Collado, J. (2012). De la lectura hipertextual a la escritura colaborativa: blogs y wikis en la didáctica de la lengua y la literatura En A. Mendoza Fillola (2012). Leer Hipertextos, Del marco hipertextual a la formación del lector literario. Barcelona: Octaedro, 262-274.

Lluch, G. (2014). Jóvenes y adolescentes hablan de lectura en la red. Ocnos, 11, 7-20. Recuperado el 1 de septiembre, 2015 de http://dx.doi.org/10.18239/ocnos 2014.11.01.

Mason, J. (2002). Researching your own practice. The discipline of noticing. London: Routledge-Falmer.

Mendoza, A. Arbonés, C. y Muñoz M.S. (2015). La proyección didáctica del hipertexto 2.0 en la comprensión, interpretación y construcción de significados de producción literaria. Lenguaje y Textos, 41, Barcelona: SEDLL-Grao, 9-18.

Rovira-Collado, José (2011a). Literatura infantil y juvenil en internet: de la Cervantes Virtual a la LIJ 2.0. Herramientas para su estudio y difusión. Ocnos, 7, 137-151. $\begin{array}{llllll}\text { Recuperado el } & 1 & \text { de } & \text { septiembre, } & 2015\end{array}$ http://dx.doi.org/10.18239/ocnos 2011.07.11.

Rovira-Collado, J. (2011b). De la competencia digital a la literaria a través de los blogs. Modelos de actuación. En Selección, desarrollo y evaluación de competencias en Didáctica de la Lengua y la Literatura Simposio Internacional de la Sociedad Española de Didáctica de la Lengua y la Literatura. Sevilla: Universidad de Sevilla, 624-641.

Rovira-Collado, J. (2015). Literatura infantil y juvenil en Internet. De la Cervantes Virtual a la LIJ 2.0. Herramientas y espacios para su estudio y difusión (Tesis doctoral) Alicante: Universidad. Recuperado el 1 de septiembre, 2015 de http://rua.ua.es/dspace/handle/10045/46345. 
Rovira-Collado J. et al. (2013). Buenas prácticas en el uso de Blogs de DLL y LIJ. Selección de modelos. En AA.VV. La producción científica y la actividad de innovación docente en proyectos de redes, Alicante: Universidad, Instituto de Ciencias de la Educación, 1556-1570.

Soria Andurell A. (2015). Hipertexto y comprensión lectora. En Investigaciones sobre Lectura ISL. 4, 51-70. Recuperado el 1 de septiembre, 2015 de http://comprensionlectora.es/revistaisl/index.php/revistaISL/article/view/52.

Van ES, E., y Sherin, M. (2002). Learning to notice: Scaffoldin new teachers intepretations of classroom interactions. Journal of Technology and Teacher Education, 10, 571-596.

Zayas, Felipe (2008). El lugar de los blogs en las áreas de lenguas, en C. Rodríguez Gonzalo, (ed). La lengua escrita y los proyectos de trabajo. Valencia, Perifèric Edicions.

Zayas, Felipe (2013). Ante una etapa de Darle a la Lengua. En Darle a la Lengua [blog]. Recuperado el 1 de septiembre, 2015 de http://www.fzayas.com/ante-una-nuevaetapa-de-darle-a-la-lengua/.

Rovira-Collado, J. (2016): Mirar como maestros para el desarrollo de la comprensión lectora. Blogs educativos para la competencia profesional en futuros docentes, Investigaciones Sobre Lectura, $6,58-75$. 


\section{ANEXO I}

Selección de 25 modelos de buenas prácticas DLL y LIJ 2.0. Universidad Alicante 2013

\begin{tabular}{|c|c|}
\hline Nombre del Blog & Dirección \\
\hline A pie de Aula & http://apiedeaula.blogspot.com.es/ \\
\hline Apuntes de Lengua & http://www.apuntesdelengua.com/blog/ \\
\hline Biblioteca Escolar & $\underline{\text { http://bibliotecaescolarinfo.blogspot.com.es }}$ \\
\hline Blog de Gemma Lluch & http://gemmalluch.com/esp/blog/ \\
\hline Blogmaniacos & http://blogmaniacosunidos.blogspot.com.es/ \\
\hline Cárcel de Papel & http://www.lacarceldepapel.com \\
\hline Cervantes Virtual & http://blog.cervantesvirtual.com/ \\
\hline Cosas profes infantil & http://cosasprofesinfantil.blogspot.com.es/ \\
\hline Crónica de los reinos & http://cronicasdelosreinos.blogspot.com.es/ \\
\hline Cuatro Gatos & http://blog.cuatrogatos.org/blog/ \\
\hline Darabuc & http://darabuc.wordpress.com/ \\
\hline Darle a la Lengua & http://www.fzayas.com/darlealalengua/ \\
\hline Ebooksinfantiles & http://ebooksinfantiles.wordpress.com/ \\
\hline Editado / infantil y juvenil & http://editadoenlij.blogspot.com.es/ \\
\hline Espacio de LIJ & http://espaciodelij.blogspot.com.es/ \\
\hline Kuentalibros & http://kuentalibros.blogspot.com.es/ \\
\hline La casa de Tomasa & http://casadetomasa.wordpress.com \\
\hline La memoria y el sol & http://lamemoriayelsol.wordpress.com/ \\
\hline Letras, libros y más & http://letraslibrosymas.blogspot.com.es/ \\
\hline LIJ Actual & http://lij-jg.blogspot.com.es/ \\
\hline Literaturas Exploratorias & http://literaturasexploratorias.tumblr.com/ \\
\hline Pizca de Papel & http://elblogdepizcadepapel.blogspot.com.es/ \\
\hline Repaso de Lengua & http://www.repasodelengua.com/ \\
\hline Soñando cuentos & http://sonandocuentos.blogspot.com.es/ \\
\hline TIC y Aprendizaje & http://mcarmendevesa.blogspot.com.es/ \\
\hline
\end{tabular}

Rovira-Collado, J. (2016): Mirar como maestros para el desarrollo de la comprensión lectora. Blogs educativos para la competencia profesional en futuros docentes, Investigaciones Sobre Lectura, $6,58-75$.
ISL, vol. 6, 2016, págs. 58-75 